\title{
Propranolol reduces systemic oxidative stress and endotoxemia in cirrhotic patients with esophageal varices
}

\author{
Dimitra Taprantzia ${ }^{a}$, Dimitrios Zisimopoulos ${ }^{b}$, Konstantinos C. Thomopoulos ${ }^{c}$, Iris Spiliopoulou ${ }^{d}$, \\ Christos D. Georgiou', Georgios Tsiaoussisc, Christos Triantos', Charalambos A. Gogos ${ }^{\mathrm{a}}$, \\ Chrisoula Labropoulou-Karatza a, Stelios F. Assimakopoulos ${ }^{a}$
}

University Hospital of Patras; University of Patras, Greece

\begin{abstract}
Background The aim of the study was to investigate the effect of propranolol on systemic oxidative stress and endotoxemia in patients with liver cirrhosis and clinically significant portal hypertension evidenced by the presence of esophageal varices.

Methods Fourteen patients with liver cirrhosis and esophageal varices, not previously been treated with non-selective beta-blockers (NSBB), were prospectively started on propranolol and followed up for three months. Serum early and late lipid peroxidation products (lipid hydroperoxides $[\mathrm{LOOH}]$ and malondialdehyde $[\mathrm{MDA}]$, respectively), and endotoxin concentrations in peripheral blood were measured. Fourteen age- and sex-matched healthy individuals were used as controls.

Results Patients with liver cirrhosis presented significantly higher systemic oxidative stress and endotoxin concentrations compared to healthy controls $(\mathrm{P}<0.001)$. Propranolol treatment for one month significantly reduced serum MDA $(\mathrm{P}<0.05)$, LOOH $(\mathrm{P}<0.01)$, and endotoxin levels $(\mathrm{P}<0.01)$ compared to pre-treatment values, whilst $\mathrm{LOOH}$ reached control levels. At three months of propranolol treatment, serum LOOH did not differ significantly from the one-month values, whilst serum endotoxin and MDA levels were further reduced between 3- and 1-month period $(\mathrm{P}<0.05$ and $\mathrm{P}<0.01$, respectively), with the latter reaching control levels. Amelioration of systemic endotoxemia at the one- and three-month follow-up intervals (compared to pre-treatment values) was not correlated with the respective reductions in serum MDA and LOOH.
\end{abstract}

Conclusions This is the first study to show that NSBB treatment in liver cirrhosis exerts a significant systemic antioxidant action. This effect seems to be, at least partly, independent of their beneficial effects on intestinal barrier function and endotoxemia.

Keywords Cirrhosis, portal hypertension, non-selective beta-blockers, lipid peroxidation, endotoxin, intestinal permeability

Ann Gastroenterol 2018; 31 (2): 1-7

\begin{abstract}
a'Department of Internal Medicine, University Hospital of Patras (Dimitra Taprantzi, Charalambos A. Gogos, Chrisoula LabropoulouKaratza, Stelios F. Assimakopoulos); ${ }^{\mathrm{b}}$ Department of Biology, University of Patras (Dimitrios Zisimopoulos, Christos D. Georgiou); 'Division of Gastroenterology, Department of Internal Medicine, University Hospital of Patras (Konstantinos C. Thomopoulos, Georgios Tsiaoussis, Christos Triantos); ${ }^{\mathrm{d} D e p a r t m e n t ~ o f ~ M i c r o b i o l o g y, ~ U n i v e r s i t y ~ H o s p i t a l ~}$ of Patras (Iris Spiliopoulou), Greece

Conflict of Interest: None
\end{abstract}

Correspondence to: Dimitra Taprantzi, MD, Department of Internal Medicine, University Hospital of Patras, Patras 26504, Greece, e-mail: dimitratap@hotmail.com

Received 27 July 2017; accepted 3 November 2017; published online 22 December 2017

DOI: https://doi.org/10.20524/aog.2017.0222

\section{Introduction}

Oxidative stress results from a persistent imbalance between the production of oxidants and cellular antioxidant systems in favor of the former; it has been recognized not only as a causative factor of cellular injury, but also as a pivotal regulator of all crucial cellular processes, including metabolism, growth, differentiation and death [1,2]. Oxidative stress has been recognized as a fundamental factor in the promotion of liver fibrogenesis and the progression of chronic liver injury into liver cirrhosis, irrespectively of its underlying etiology $[3,4]$. Most importantly, oxidative stress seems to possess a systemic spread in cirrhosis playing a pivotal pathophysiological role in the structural and/or functional derangements developed in diverse organs (intestinal barrier dysfunction, spontaneous bacterial peritonitis, portal hypertension-endothelial 
dysfunction-hyperdynamic circulation, hepatorenal and hepatopulmonary syndromes, hepatic encephalopathy and cardiomyopathy, hepatocellular carcinoma) [5].

The presence of systemic endotoxemia, promoted by failure of the intestinal barrier in liver cirrhosis, has been widely accepted as an important common pathogenetic mechanism in the development of diverse complications of cirrhosis. Endotoxemia exerts its systemic injurious effects through activation of the leukocyte system, which produces and releases numerous inflammatory and vasoactive mediators and oxygen free radicals [6-8]. Portal hypertension has previously been shown to be strictly correlated with intestinal hyperpermeability and also constitutes the pathophysiological basis of most complications of cirrhosis [9].

Non-selective beta-blockers (NSBB), such as propranolol, have been long used for the pharmacological treatment of cirrhotic portal hypertension in order to prevent the first episode or the recurrence of variceal bleeding [10]. Additionally, recent meta-analyses have shown that NSBB decrease mortality in cirrhotic patients to a greater degree than would be expected from the reduced rate of variceal bleeding $[11,12]$, and also protect against spontaneous bacterial peritonitis [13]. Notably, a recent important clinical study demonstrated their beneficial effects in the prevention of gut-derived endotoxemia and its associated systemic cytokinemia in cirrhosis [9]. Endotoxemia and its associated systemic inflammatory response are wellestablished promoters of systemic oxidative stress in liver cirrhosis [5]. However, to the best of our knowledge, there are no previous studies investigating the effect of NSBB therapy on this important pathophysiological factor for the development of complications of cirrhosis.

The present study was undertaken to investigate the effect of propranolol treatment on serum oxidative stress and endotoxemia in patients with liver cirrhosis and clinically significant portal hypertension, determined by the presence of esophageal varices.

\section{Patients and methods}

\section{Blood collection and storage}

Blood samples of healthy donors and cirrhotic patients were collected to measure endotoxin, lipid hydroperoxides (LOOH) and malondialdehyde (MDA). Blood was withdrawn from a peripheral vein using pyrogen-free syringes and collected in endotoxin-free vials. The serum was separated from the cells by centrifugation at $4000 \mathrm{rpm}$ for $15 \mathrm{~min}$ and stored at $-80^{\circ} \mathrm{C}$ until analysis.

\section{Patients}

The study was approved by the Ethics Committee of Patras University Hospital, Patras, Greece. All subjects were informed about the study protocol and written informed consent was obtained prior to their enrollment in the study.

Fourteen patients with liver cirrhosis and esophageal varices not currently treated with a NSSB were prospectively enrolled in this study. Patients with small varices were treated with NSSB, either because according to endoscopy findings there were red wale signs on varices, or because their cirrhosis severity was Child-Pugh C (following the guidelines of the American Association for the Study of Liver Diseases). The diagnosis of liver cirrhosis was established by means of histology and/or by its clinical, laboratory, endoscopic, or imaging manifestations. Cirrhotic patients were enrolled after being diagnosed with esophageal varices on upper gastrointestinal endoscopy performed for diagnostic reasons or 7-10 days after an episode of variceal bleeding, managed endoscopically with variceal ligation, when the gastrointestinal bleeding had ceased and the patient was hemodynamically stable. Additionally, antibiotics were used in patients with variceal bleeding only for a few days as prophylactic therapy. According to the pharmacokinetics of the antibiotics used in variceal bleeding, the average elimination half-life of the antibiotics used in the patients of our study with variceal bleeding was less than a day. In these patients samples were taken 7-10 days after cessation of variceal bleeding and a minimum of 2 days after cessation of antibiotic therapy. In patients with liver cirrhosis due to alcohol consumption, alcohol had been discontinued at least one year before the initiation of the study. Cirrhosis severity was assessed according to the Child-Pugh classification. Patients with acute liver failure, hepatic encephalopathy, hepatorenal syndrome, hepatoma or other malignancy, alcohol abuse in the previous 4 weeks, infections including spontaneous bacterial peritonitis, rheumatic diseases, renal diseases, gastrointestinal diseases (e.g., celiac disease, inflammatory bowel disease) or intestinal surgery, under treatment with corticosteroids, nonsteroid anti-inflammatory drugs or antioxidants (vitamins $\mathrm{C}$ and E, allopurinol, N-acetyl-cysteine), history of surgery or transjugular intrahepatic portosystemic shunt for portal hypertension, history of use of NSSB medication, or patients with contraindications to NSSB were excluded. The propranolol dose was titrated aiming at a heart rate of 55-60 bpm (adjusted doses ranged from 40 to $240 \mathrm{mg}$ per day, administered in two or three divided doses). The patients were followed up for a three-month period (before starting propranolol, one-month, and three-months after starting propranolol treatment).

The control group comprised healthy individuals matched for age and sex ( $\mathrm{n}=14,9$ male/5 female), with no past medical history, normal clinical examination and laboratory blood tests.

\section{Oxidative stress assessment}

Oxidative stress evaluation was performed by measuring two main indicators of lipid peroxidation damage, the early and late lipid peroxidation products $\mathrm{LOOH}$ and MDA, respectively [14], in blood serum, as follows. For the $\mathrm{LOOH}$ assay, a $0.6 \mathrm{~mL}$ sample was diluted with $0.6 \mathrm{~mL}$ phosphate-BHA buffer (10 mM phosphate buffer, $\mathrm{pH} 7.2$, containing $0.5 \mathrm{mM}$ BHA, from 0.33 M BHA stock, made in absolute ethanol, to 
prevent artificial lipid peroxidation during the assay), mixed by vortexing with $1.2 \mathrm{~mL}$ chloroform: methanol (2:1 v:v) and $0.18 \mathrm{~mL} 100 \% \mathrm{TCA}$, and kept in an ice-water bath for $20 \mathrm{~min}$. The chloroform fraction containing $\mathrm{LOOH}$ was separated by centrifugation at $15000 \mathrm{~g}$ for $5 \mathrm{~min}$, vacuum-dried, and the resulting lipid pellet was solubilized in $1 \mathrm{~mL}$ methanol. Estimation of LOOH was carried out with FOX assay and the result was expressed as equivalents of nmoles of cumene hydroperoxide per $\mathrm{mL}$ serum. For the total (free plus proteinbound) MDA assay, a $0.02 \mathrm{~mL}$ sample was diluted to $0.25 \mathrm{~mL}$ with phosphate-BHA buffer, and mixed with $0.05 \mathrm{~mL}$ TBA reagent and $3 \mu \mathrm{L} 0.1 \mathrm{M} \mathrm{BHA}$ (made in absolute ethanol). The mixture was incubated at $100^{\circ} \mathrm{C}$ for $20 \mathrm{~min}$, brought to room temperature, vortexed with $0.3 \mathrm{~mL}$ butanol-1 and centrifuged at $15000 \mathrm{~g}$ for $3 \mathrm{~min}$. The fluorescence of the upper butanol layer was measured at ex/em 535/550 nm. MDA was expressed as nmoles per $\mathrm{mL}$ serum. $\mathrm{LOOH}$ and MDA have been used in studies as markers of oxidative stress damage in liver cirrhosis $[15,16]$.

\section{Endotoxin measurements}

Blood samples of healthy donors and patients were collected in endotoxin-free vials. After centrifugation, serum was separated and stored at $-80^{\circ} \mathrm{C}$ until processed. Endotoxin levels were measured by the quantitative chromogenic Limulus Amebocyte Lysate (LAL) test (QCL-100; Lonza, Walkersville, $\mathrm{MD}$, USA), according to the manufacturer's instructions. Briefly, the stored samples were defrosted at room temperature and four standard endotoxin solutions were prepared. Then $50 \mu \mathrm{L}$ of each sample was mixed with the LAL, added to the microplate well and incubated at $37^{\circ} \mathrm{C}$ for $10 \mathrm{~min}$. The LALsample was mixed with a chromagen, incubated at $37^{\circ} \mathrm{C}$ for an additional $6 \mathrm{~min}$ after which the reaction was stopped by a stop reagent. The absorbance of the sample, determined spectrophotometrically, is in direct proportion to the amount of endotoxin present, and thus, the concentration of endotoxin can be calculated from a standard curve created by the absorbance of standard endotoxin solutions. The endotoxin concentrations were expressed as EU/mL. All materials used in this test were endotoxin-free.

\section{Statistical analysis}

Statistical analysis was performed using the SPSS statistical package (version 20; SPSS, Chicago, IL, USA). Normality of data was tested using the Shapiro-Wilk test. As the distributions of MDA, LOOH and endotoxin were non-normal, Friedman analysis with post hoc Wilcoxon signed-rank tests were performed. Comparisons between controls and cirrhotics at diverse time points were made using the Mann-Whitney $U$-test. Correlation analyses were performed using the nonparametric Spearman correlation test. In all cases a P-value of $<0.05$ was considered as significant.

\section{Results}

\section{Patient characteristics}

Characteristics of the enrolled population, before the initiation of propranolol treatment, are summarized in Table 1. Briefly, 14 patients with liver cirrhosis and 14 healthy controls were involved in the study. No significant differences with regard to age or sex were observed among cirrhotic patients and controls. Alcoholic liver disease and viral hepatitis were the main etiologies of cirrhosis in the studied sample, which also included two patients with unknown etiology. Nine patients had compensated cirrhosis classified as Child-Pugh A, whereas five patients had decompensated cirrhosis classified as ChildPugh Class B or $C$ ( $n=3$ and $n=2$, respectively). All cirrhotic patients involved in this study presented with esophageal varices of varying size. Three patients with variceal bleeding were included in the study 7-10 days after the cessation of bleeding.

\section{Levels of serum oxidative stress markers}

Before treatment with a NSSB, cirrhotic patients presented significantly higher levels of serum MDA and LOOH as compared to healthy controls $(\mathrm{P}<0.001$ for both) (Fig. 1 and Table 2). After one month of propranolol treatment, there was a significant decline in both MDA and $\mathrm{LOOH}$ levels as compared to pre-

Table 1 Characteristics of patients with liver cirrhosis before the initiation of propranolol treatment (time: 0 ) and healthy controls

\begin{tabular}{|c|c|c|}
\hline Characteristics & $\begin{array}{l}\text { Cirrhotic patients } \\
\text { (time: } 0)(\mathrm{n}=14)\end{array}$ & $\begin{array}{c}\text { Healthy } \\
\text { controls }(\mathrm{n}=14)\end{array}$ \\
\hline Age & $67(42-79)$ & $63(38-80)$ \\
\hline Sex & $10 \mathrm{M} / 4 \mathrm{~F}$ & $9 \mathrm{M} / 5 \mathrm{~F}$ \\
\hline $\begin{array}{l}\text { Etiology of cirrhosis } \\
\text { (alcohol/viral/ } \\
\text { unknown) }\end{array}$ & $6 / 6 / 2$ & - \\
\hline $\begin{array}{l}\text { Compensated/ } \\
\text { decompensated }\end{array}$ & $9 / 5$ & - \\
\hline $\begin{array}{l}\text { Child-Pugh class } \\
(\mathrm{A} / \mathrm{B} / \mathrm{C})\end{array}$ & $9 / 3 / 2$ & - \\
\hline INR & $1.17(0.95-1.68)^{*}$ & $0.99(0.68-1.37)$ \\
\hline Albumin (g/dL) & $3.65(2.30-5.00)^{*}$ & $4.50(3.60-4.80)$ \\
\hline Total bilirubin (mg/dL) & $1.35(0.30-6.91)^{*}$ & $0.45(0.30-0.70)$ \\
\hline Ascites & $3 / 14$ & - \\
\hline Esophageal varices & $14 / 14$ & - \\
\hline $\begin{array}{l}\text { Esophageal varices } \\
\text { grade } \\
\text { (small/medium/ large) }\end{array}$ & $8 / 2 / 4$ & - \\
\hline $\begin{array}{l}\text { Recent variceal } \\
\text { bleeding }\end{array}$ & $3 / 14$ & - \\
\hline
\end{tabular}


treatment values $(\mathrm{P}<0.05$ and $\mathrm{P}<0.01$, respectively) (Fig. 1,2$)$ and $\mathrm{LOOH}$ was reduced to healthy control levels. At three months of propranolol treatment, $\mathrm{LOOH}$ remained at control levels, whilst MDA was further reduced as compared to the one-month values $(\mathrm{P}<0.01)$ and also reached control levels (Figs. 1, 2 and Table 2).

\section{Serum endotoxin concentrations}

Before the initiation of propranolol treatment, patients with liver cirrhosis presented higher serum endotoxin levels as compared to healthy controls $(\mathrm{P}<0.001$, Fig. 1 and Table 2$)$. In patients with variceal bleeding, blood samples were taken 7-10 days after the cessation of variceal bleeding when endotoxin values were no longer influenced by the bleeding [17].

Systemic endotoxemia was significantly ameliorated after one month of propranolol treatment $(\mathrm{P}<0.01$ vs. pre-treatment values) and further significantly reduced at three months ( $\mathrm{P}<0.05$ vs. one-month values) (Figs. 1,2$)$. However, at both time points after initiation of propranolol treatment, endotoxin levels were significantly higher than those of healthy controls $(\mathrm{P}<0.001)$, as shown in Fig. 1 and Table 2.

Table 2 Serum MDA, LOOH and endotoxin levels

\begin{tabular}{|c|c|c|c|c|c|c|}
\hline Serum & $\begin{array}{l}\text { Group I } \\
\mathrm{n}=14\end{array}$ & $\begin{array}{l}\text { Group II } \\
\mathrm{n}=14\end{array}$ & $\begin{array}{l}\text { Group III } \\
\mathrm{n}=14\end{array}$ & $\begin{array}{l}\text { Group IV } \\
\mathrm{n}=14\end{array}$ & $\begin{array}{c}\text { P-value } \\
\text { (Control/ } \\
\text { Patients at time } \\
\text { points) }\end{array}$ & $\begin{array}{c}\text { P-value } \\
\text { (Patients at different } \\
\text { time points) }\end{array}$ \\
\hline $\begin{array}{l}\text { MDA } \\
\text { (nmoles } / \mathrm{mL})\end{array}$ & $\begin{array}{c}0.30 \\
(0.26-0.34)\end{array}$ & $\begin{array}{c}0.38 \\
(0.21-0.73)\end{array}$ & $\begin{array}{c}0.37 \\
(0.21-0.46)\end{array}$ & $\begin{array}{c}0.26 \\
(0.18-0.39)\end{array}$ & $\begin{array}{l}\text { I/II:0.00, I/III:0.022, } \\
\text { I/IV: NS, }\end{array}$ & $\begin{array}{c}\text { II/III: } 0.044 \\
\text { II/IV: } 0.001, \text { III/IV:0.001 }\end{array}$ \\
\hline $\begin{array}{l}\text { LOOH } \\
\text { (nmoles cumene } \\
\text { equivalents } / \mathrm{mL} \text { ) }\end{array}$ & $\begin{array}{c}2.84 \\
(1.87-3.52)\end{array}$ & $\begin{array}{c}4.79 \\
(2.78-10.88)\end{array}$ & $\begin{array}{c}3.43 \\
(2.13-6.44)\end{array}$ & $\begin{array}{c}2.84 \\
(1.95-6.00)\end{array}$ & $\begin{array}{c}\text { I/II: 0.000, I/III: NS, } \\
\text { I/IV: NS, }\end{array}$ & $\begin{array}{c}\text { II/III:0.005, II/IV:0.008, } \\
\text { III/IV: NS }\end{array}$ \\
\hline $\begin{array}{l}\text { Endotoxin } \\
(\mathrm{EU} / \mathrm{mL})\end{array}$ & $\begin{array}{c}0.27 \\
(0.23-0.37)\end{array}$ & $\begin{array}{c}0.52 \\
(0.35-0.90)\end{array}$ & $\begin{array}{c}0.42 \\
(0.33-0.73)\end{array}$ & $\begin{array}{c}0.42 \\
(0.30-0.57)\end{array}$ & $\begin{array}{c}\text { I/II:0.000, I/III:0.000, } \\
\text { I/IV:0.000, }\end{array}$ & $\begin{array}{c}\text { II/III:0.003, II/IV:0.005, } \\
\text { III/IV:0.022 }\end{array}$ \\
\hline
\end{tabular}

Values are medians (min-max), Group I: Control, Group II: Cirrhotic patients (time: 0), Group III: Cirrhotic patients (time: 1 month after the initiation of propranolol), Group IV: Cirrhotic patients (time: 3 months after the initiation of propranolol)

LOOH, lipid hydroperoxides; MDA, malondialdehyde; NS, not significant

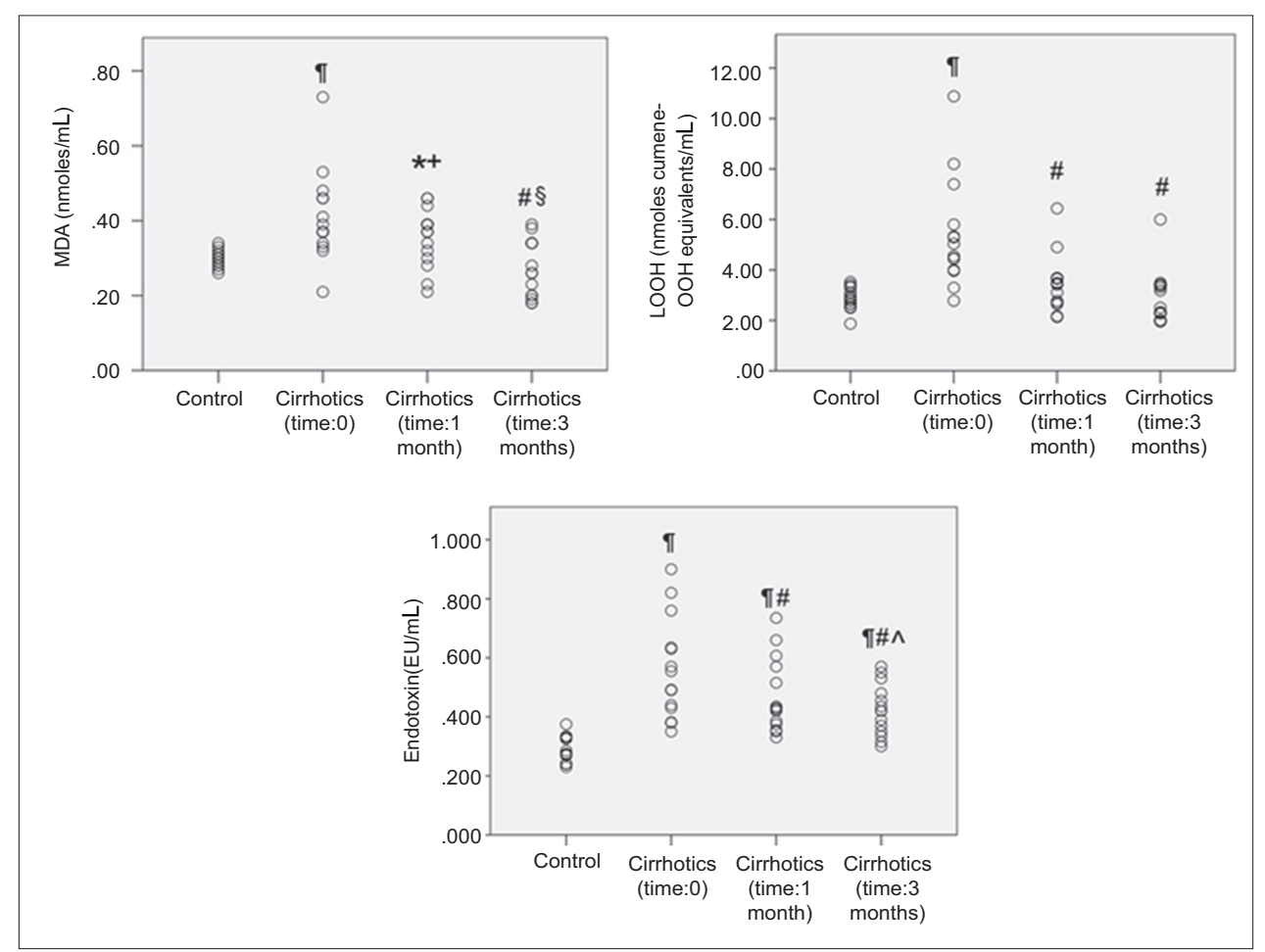

Figure 1 Dot-plot graphs showing serum MDA, LOOH and endotoxin concentrations (all data for each group)

${ }^{\star} \mathrm{P}<0.05$ vs. Control, $\mathbf{g} \mathrm{P}<0.001$ vs. Control, $+\mathrm{P}<0.05$ vs. Cirrhotics (time $=0$ ), $\# \mathrm{P}<0.01$ vs. Cirrhotics (time $=0$ ), $\wedge \mathrm{P}<0.05$ vs. Cirrhotics (time $=1$ month), $₫ \mathrm{P}<0.01$ vs. Cirrhotics (time $=1$ month). Time indicates the months after the initiation of propranolol treatment in cirrhotic patients $\mathrm{LOOH}$, lipid hydroperoxides; $M D A$, malondialdehyde 


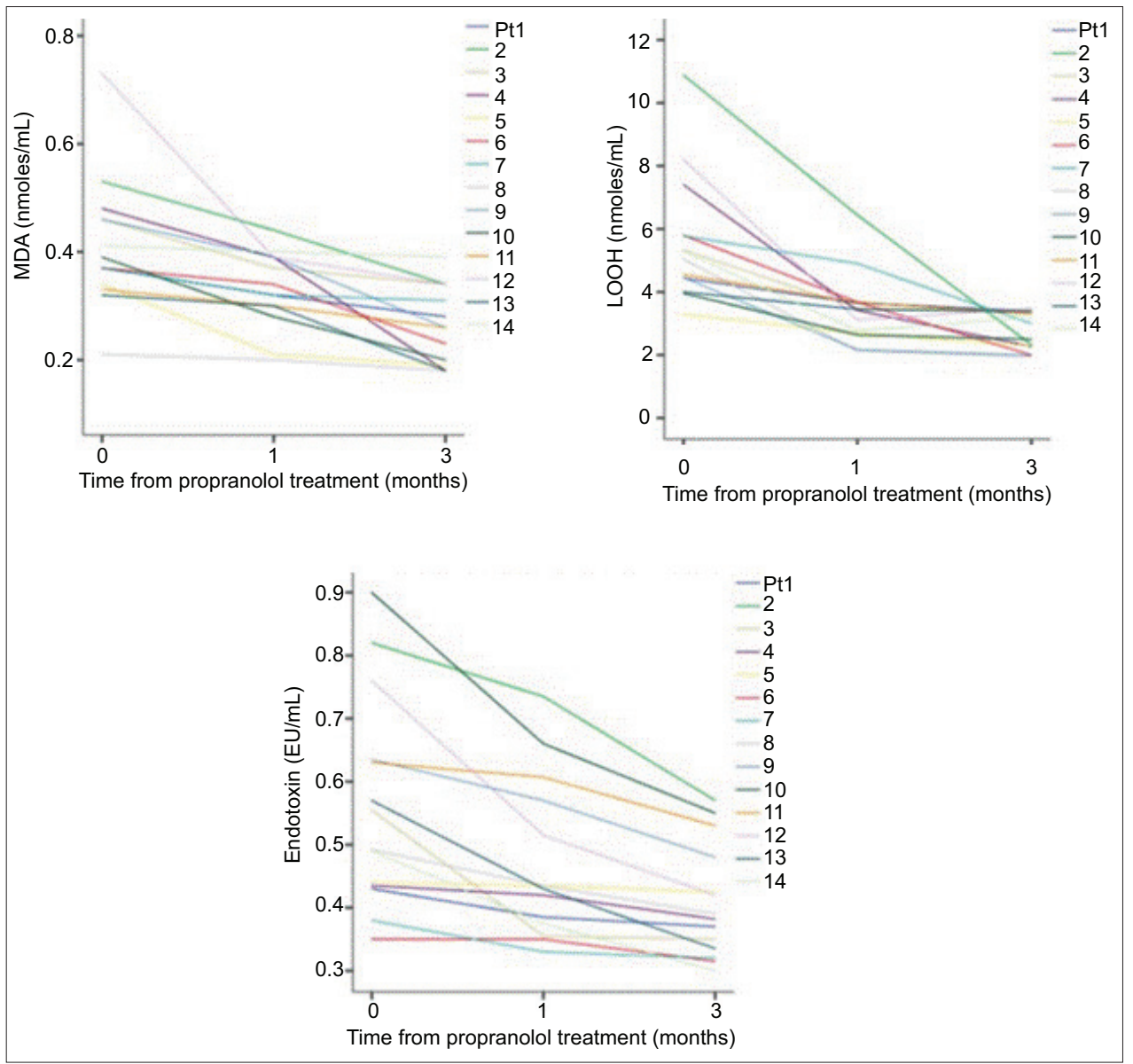

Figure 2 Line diagrams showing changes in serum MDA, LOOH and endotoxin concentrations $L O O H$, lipid hydroperoxides; MDA, malondialdehyde

\section{Correlations}

In cirrhotic patients, reduction of serum oxidative stress parameters (MDA or LOOH) after one or three months of propranolol treatment was not correlated with the respective reductions of serum endotoxin values compared to pretreatment values.

In the total population of cirrhotic patients (pooled patients with decompensated and compensated liver cirrhosis), levels of serum endotoxin and MDA levels significantly correlated with the presence of ascites $(r=0.583, \mathrm{P}=0.029$; and $r=0.651$, $\mathrm{P}=0.012$ for endotoxin and MDA, respectively). Furthermore, MDA levels significantly correlated with the cirrhosis status (decompensated vs. compensated: $r=0.614, \mathrm{P}=0.020$ ), the size of esophageal varices (small/medium/large: $r=0.581, \mathrm{P}=0.029)$ and the Child-Pugh score (A/B/C: $r=0.670, \mathrm{P}=0.009)$.

\section{Discussion}

Oxidative stress seems to play a fundamental role in hepatic cirrhosis and its complications from diverse organs [5].
Previous clinical studies in cirrhosis have demonstrated a high oxidative stress in the blood and liver tissue and we have also recently detected high lipid peroxidation levels in the intestinal mucosa of cirrhotic patients $[4,18,19]$. The role of oxidative stress in the development of cirrhosis-related complications from diverse organs has been emphasized previously $[5,20]$. The systemic spread of oxidative stress in liver cirrhosis points towards its potential importance as a pharmacological target.

Despite its indisputably important pathophysiological role in the development of complications of cirrhosis, the effect of diverse pharmacological modalities used to address this factor in patients with cirrhosis has not been adequately investigated. NSBB constitute the cornerstone pharmacological therapy for the control of portal hypertension for the primary or secondary prevention of variceal bleeding. To the best of our knowledge, this is the first study investigating in humans the effect of NSBB on systemic oxidative stress in liver cirrhosis.

According to our findings, propranolol effectively reduced serum levels of both oxidative stress markers (MDA and $\mathrm{LOOH}$ ) from the first month after treatment initiation. More specifically, $\mathrm{LOOH}$ was restored to control levels even from the first month of propranolol treatment and this positive effect was sustained at three months, whilst MDA was significantly 
reduced at first month and reached control levels at three months. This more delayed response of MDA to propranolol treatment might be due to the fact that, since $\mathrm{LOOH}$ are the early decomposition products of peroxidized lipids, there is a time lag between their initial emergence and the subsequent decomposition to MDA of those $\mathrm{LOOH}$ that have escaped the antioxidant defense of the organism. Concurrently, NSBB therapy significantly ameliorated systemic endotoxemia at the one- and three-month follow-up intervals, but endotoxemia prevention was not correlated with the respective reductions of serum MDA and LOOH. An antioxidant action of NSBB has been previously demonstrated in myocardial infraction and cardiac failure [20-23]. In liver cirrhosis, oxidative stress is mainly promoted by the presence of gut-derived endotoxemia in the systemic circulation, which activates the leukocyte system to produce and release inflammatory mediators and oxygen free radicals $[5,19]$. Prevention of systemic endotoxemia by NSBB therapy, through improvement of intestinal permeability, would have been a reasonable explanation for the observed reduction in systemic oxidative stress. However, in the present study propranolol-induced oxidative stress reduction was not correlated with the respective amelioration of systemic endotoxemia. This finding implies that additional or diverse mechanisms underlie the antioxidant effect of NSBB in cirrhosis.

Previous studies have shown that propranolol may attenuate oxidative stress through blockade of the catecholamine-induced activation of the hepatic endoplasmic reticulum stress [24]. In addition, it has been previously shown that propranolol may protect cells against oxidative stress through primary antiradical properties independent of beta-receptor blockade [25]. Propranolol's antioxidant properties are in part linked to the protection of membranes against lipid peroxidation [26]. Propranolol reduces intracellular $\mathrm{Ca}^{++}$overload and attenuates mitochondrial dysfunction by its action on electron mitochondrial membrane transport, and also by inhibiting the Bax-mediated cytochrome C release [20,27]. Interestingly an experimental study in rats with $\mathrm{CCl} 4$ induced hepatotoxicity confirmed the antioxidant effects of NSSB. More specifically, the study showed that carvedilol, an NSBB, showed promising antifibrotic effects by reducing the oxidative stress [28].

In agreement with previous reports, the present study also demonstrated a positive impact of propranolol on intestinal barrier function and endotoxemia. A recent study showed that the positive effect of propranolol on the amelioration of intestinal hyperpermeability in cirrhosis is partially independent of its hemodynamic effects on portal pressure [9]. Our present findings demonstrate that improvement in barrier function by propranolol treatment is also independent of its antioxidant action, despite the fact that oxidative stress seems to be an important factor contributing to intestinal injury in cirrhosis [19]. Alternative explanations for the observed prevention of gut-derived endotoxemia by propranolol might be: (a) the acceleration of the intestinal transit time leading to prevention of gut bacterial overgrowth and consequently of intraluminal endotoxin load [29]; (b) the inhibition, via the splanchnic sympathetic nervous system, of the detrimental immunosuppressive effects of cirrhosis-induced sympathetic overactivity on the innate immune system [30]; and (c) the potential restoration of intestinal tight junction integrity [31].

In conclusion, this is the first clinical study to demonstrate that propranolol exerts a systemic antioxidant action, concurrently with endotoxemia prevention, in cirrhotic patients with esophageal varices. Although the number of patients included in the present study was limited, our findings might be of clinical significance, considering the pivotal pathophysiological role of endotoxemia and oxidative stress in complications of cirrhosis from diverse organs [5]. Previous studies have shown that NSBB treatment reduces the incidence of hepatocellular carcinoma and spontaneous bacterial peritonitis occurrence in cirrhotic patients [32], which might be associated with their systemic antioxidant action $[5,11]$; however, other investigations have contradicted these findings [33]. Beyond their well-established preventive role in variceal bleeding, we feel that future studies should focus on answering whether the NSBB-induced systemic antioxidant effect and gut barrier function improvement could result in the prevention of other cirrhosis-related complications, leading to a better clinical outcome in terms of morbidity and mortality.

\section{Summary Box}

\section{What is already known:}

- In liver cirrhosis, gut barrier failure leads to portal and systemic endotoxemia with subsequent activation of a systemic inflammatory and prooxidant response

- Systemic oxidative stress and endotoxemia play a fundamental pathophysiological role in the aggravation of liver injury and the development of complications of cirrhosis from diverse organs

- Non-selective beta-blockers (NSSB) reduce intestinal hyperpermeability, bacterial translocation and endotoxemia, independently of their hemodynamic effects on portal pressure, whilst a decreased incidence of spontaneous bacterial peritonitis and hepatocellular carcinoma has been also reported

\section{What the new findings are:}

- Propranolol exerts a systemic antioxidant action in liver cirrhosis, concurrently with endotoxemia prevention, in cirrhotic patients with esophageal varices

- The systemic antioxidant effects of NSBB are related to additional mechanisms apart from the improvement in intestinal barrier function and amelioration of endotoxemia, since endotoxemia prevention and antioxidant effect were not correlated in our study 


\section{Acknowledgment}

This study was supported by the "Andreas Mentzelopoulos Scholarships for Studies in the University of Patras" (Dimitra Taprantzi, is the scholarship recipient).

\section{References}

1. Halliwell B, Gutteridge J. Free Radicals in biology and medicine. OUP Oxford 2008.

2. Buttke TM, Sandstrom PA. Oxidative stress as a mediator of apoptosis. Immunol Today 1994;15:7-10.

3. Tanikawa K, Torimura T. Studies on oxidative stress in liver diseases: important future trends in liver research. Med $\mathrm{Mol}$ Morphol 2006;39:22-27.

4. Moustafa AH, Ali EM, Mohamed TM, Abdou HI. Oxidative stress and thyroid hormones in patients with liver diseases. Eur J Intern Med 2009;20:703-708.

5. Assimakopoulos SF, Gogos C, Labropoulou-Karatza C. Could antioxidants be the "magic pill" for cirrhosis-related complications? A pathophysiological appraisal. Med Hypotheses 2011;77:419-423.

6. Kalambokis G, Tsianos EV. Endotoxaemia in the pathogenesis of cytopenias in liver cirrhosis. Could oral antibiotics raise blood counts? Med Hypotheses 2011;76:105-109.

7. Nolan JP. The role of intestinal endotoxin in liver injury: a long and evolving history. Hepatology 2010;52:1829-1835.

8. Cavasin MA, Semus H, Pitts K, et al. Acute effects of endothelin receptor antagonists on hepatic hemodynamics of cirrhotic and noncirrhotic rats. Can J Physiol Pharmacol 2010;88:636-643.

9. Reiberger T, Ferlitsch A, Payer BA, et al; Vienna Hepatic Hemodynamic Lab. Non-selective betablocker therapy decreases intestinal permeability and serum levels of LBP and IL-6 in patients with cirrhosis. J Hepatol 2013;58:911-921.

10. Garcia-Tsao G, Sanyal AJ, Grace ND, Carey W; Practice Parameters Committee of the American College of Gastroenterology. Prevention and management of gastroesophageal varices and variceal hemorrhage in cirrhosis. Hepatology 2007;46:922-938.

11. Krag A, Wiest R, Albillos A, Gluud LL. The window hypothesis: haemodynamic and non-haemodynamic effects of $\beta$-blockers improve survival of patients with cirrhosis during a window in the disease. Gut 2012;61:967-969.

12. Krag A, Wiest R, Gluud LL. Reduced mortality with non-selective beta blockers compared to banding is not related to prevention of bleeding or bleeding related mortality: systematic review of randomized trials. J Hepatol 2011;54 Suppl 1:S72.

13. Senzolo M, Cholongitas E, Burra $\mathrm{P}$, et al. $\beta$-blockers protect against spontaneous bacterial peritonitis in cirrhotic patients: a metaanalysis. Liver Int 2009;29:1189-1193.

14. Grintzalis K, Zisimopoulos D, Grune T, Weber D, Georgiou CD. Method for the simultaneous determination of free/ protein malondialdehyde and lipid/protein hydroperoxides. Free Radic Biol Med 2013;59:27-35.

15. Arauz J, Ramos-Tovar E, Muriel P. Redox state and methods to evaluate oxidative stress in liver damage: From bench to bedside. Ann Hepatol 2016;15:160-173.

16. Clot $\mathrm{P}$, Tabone $\mathrm{M}$, Aricò S, Albano E. Monitoring oxidative damage in patients with liver cirrhosis and different daily alcohol intake. Gut 1994;35:1637-1643.

17. Garbuzenko D, Mikurov A, Smirnov D. The bacterial endotoxins levels in the blood of cirrhotic patients as predictor of the risk of esophageal varices bleeding. In: Garbuzenko D (Ed.), Portal hypertension - causes and complications. InTech 2012.

18. Czeczot H, Scibior D, Skrzycki M, Podsiad M. Glutathione and GSH-dependent enzymes in patients with liver cirrhosis and hepatocellular carcinoma. Acta Biochim Pol 2006;53:237-242.

19. Assimakopoulos SF, Tsamandas AC, Tsiaoussis GI, et al. Intestinal mucosal proliferation, apoptosis and oxidative stress in patients with liver cirrhosis. Ann Hepatol 2013;12:301-307.

20. Mansuy P, Mougenot N, Ramirez-Gil JF, et al. Effects of prolonged propranolol treatment on left ventricular remodeling and oxidative stress after myocardial infarction in rats. J Cardiovasc Pharmacol 2000;35:806-813.

21. Mak IT, Weglicki WB. Potent antioxidant properties of 4-Hydroxylpropranolol. J Pharmac Exp Ther 2004;308:85-90.

22. Kramer JH, Spurney CF, Iantorno M, et al. d-Propranolol protects against oxidative stress and progressive cardiac dysfunction in iron overloaded rats. Can J Physiol Pharmacol 2012;90:1257-1268.

23. Nakamura K, Kusano K, Nakamura Y, et al. Carvedilol decreases elevated oxidative stress in human failing myocardium. Circulation 2002; 105:2867-2871.

24. Brooks NC, Song J, Boehning D, et al. Propranolol improves impaired hepatic phosphatidylinositol 3-kinase/akt signaling after burn injury. Mol Med 2012;18:707-711.

25. Kramer JH, Mak IT, Freedman AM, Weglicki WB. Propranolol reduces anoxia/reoxygenation-mediated injury of adult myocytes through an anti-radical mechanism. J Mol Cell Cardiol 1991;23:1231-1244.

26. Pearce PC, Hawkey CM, Symons C, EG O. The importance of membrane stabilization in protecting the developing rat myocardium from the actions of triac. Am J Cardiovasc Pathol 1988;2:173-179.

27. Fiskum G, Starkov A, Polster BM, Chinopoulos C. Mitochondrial mechanisms of neural cell death and neuroprotective interventions in Parkinson's disease. Ann N Y Acad Sci 2003;991:111-119.

28. Hamdy N, El-Demerdash E. New therapeutic aspect for carvedilol: antifibrotic effects of carvedilol in chronic carbon tetrachloride-induced liver damage. Toxicol Appl Pharmacol 2012;261:292-299.

29. Pérez-Paramo M, Muñoz J, Albillos A, et al. Effect of propranolol on the factors promoting bacterial translocation in cirrhotic rats with ascites. Hepatology 2000;31:43-48.

30. Worlicek M, Knebel K, Linde HJ, et al. Splanchnic sympathectomy prevents translocation and spreading of $\mathrm{E}$ coli but not $\mathrm{S}$ aureus in liver cirrhosis. Gut 2010;59:1127-1134.

31. Assimakopoulos SF, Tsamandas AC, Tsiaoussis GI, et al. Altered intestinal tight junctions' expression in patients with liver cirrhosis: a pathogenetic mechanism of intestinal hyperpermeability. Eur $J$ Clin Invest 2012;42:439-446.

32. Nkontchou G, Aout M, Mahmoudi A, et al. Effect of long-term propranolol treatment on hepatocellular carcinoma incidence in patients with HCV-associated cirrhosis. Cancer Prev Res (Phila) 2012;5:1007-1014.

33. Kim TW, Kim HJ, Chon CU, et al. Is there any vindication for low dose nonselective $\beta$-blocker medication in patients with liver cirrhosis? Clin Mol Hepatol 2012;18:203-212. 